\title{
Effective medium theory for magnetodielectric composites: Beyond the long-wavelength limit
}

\author{
Ying $\mathrm{Wu}$, Jensen Li, Zhao-Qing Zhang, and C. T. Chan \\ Department of Physics, Hong Kong University of Science and Technology, Clear Water Bay, Kowloon, Hong Kong, China
}

(Received 24 April 2006; published 15 August 2006)

\begin{abstract}
We present an effective medium theory that simultaneously determines the effective permittivity and effective permeability of a magnetodielectric composite beyond the long-wavelength limit. In the long-wavelength limit, our theory reproduces previous results obtained in two and three dimensions. To verify our theory, we performed band-structure calculations on a periodic composite in two dimensions for a specific configuration with complicated Mie resonances in the microstructure. Our results show that our theory can be valid even when the wavelength in the background medium, $\lambda_{0}$, is as small as 1.3 times the lattice constant, $a$, whereas the long-wavelength theory breaks down when $\lambda_{0}<3.5 a$
\end{abstract}

DOI: 10.1103/PhysRevB.74.085111

PACS number(s): 41.20.Jb, 42.70.Qs, 78.20.Ci

\section{INTRODUCTION}

Recently, electromagnetic negative refractive index materials ${ }^{1}$ with simultaneously negative effective permittivity $\left(\varepsilon_{\text {eff }}\right)$ and permeability $\left(\mu_{\text {eff }}\right)$ have attracted tremendous interest due to their successful fabrication ${ }^{2}$ and the demonstration of novel phenomena such as subwavelength focusing. ${ }^{3}$ The fabricated system consists of a periodic array of conducting nonmagnetic split ring resonators and conducting thin wires. It has been successfully demonstrated that, in a frequency region above the electromagnetic resonances of the microstructures, the material behaves like a homogeneous medium capable of producing "double negativity" (negative $\varepsilon_{\text {eff }}$ and $\mu_{\text {eff }}$ ), and its electromagnetic properties have been described using effective medium approaches. ${ }^{4,5}$ Other systems have been proposed to produce both negative $\varepsilon_{\text {eff }}$ and negative $\mu_{\text {eff }}$, such as metallic magnetic granular composites $^{6}$ and granular composites with large permittivity and permeability in the grains. ${ }^{7}$ A common feature of all these materials is that their microstructures contain complicated electric and magnetic resonances.

In order to give a simple description of wave propagation in these materials, it is important to establish a twoparameter effective medium theory for both effective permittivity and effective permeability that can work at frequencies near or above the intrinsic resonances of the microstructures. Various effective medium theories have been proposed in the past. ${ }^{6,8-18}$ It has long been proposed that, at finite frequencies, the effective permittivity in composites could be defined by the ratio of the Fourier component of the displacement field to that of the electric field, i.e., $\varepsilon_{\text {eff }}(\omega)$ $=\vec{D}\left(k_{\mathrm{eff}}, \omega\right) / \vec{E}\left(k_{\mathrm{eff}}, \omega\right)$, where $k_{\mathrm{eff}}$ is the wave vector in the effective medium. ${ }^{8}$ By generalizing this definition to effective permeability, Chui $e t a l$. found a single equation for $k_{\mathrm{eff}}$, or $(\sqrt{\varepsilon} \sqrt{\mu})_{\text {eff }}$, for metamaterials. ${ }^{6}$ Although this approach does not necessarily require a small value of $k_{\mathrm{eff}} r_{0}$, where $r_{0}$ is the linear size of the microstructure, the effective impedance, $(\sqrt{\mu} / \sqrt{\varepsilon})_{\text {eff }}$, cannot be determined from this approach. ${ }^{6}$ A different approach is to consider the scattering problem of a coated particle in an effective medium. The coated particle consists of a granular particle of inner radius $r_{s}$ and a layer of background medium with an outer radius $r_{0}$. The effective medium is determined by the condition that total scattering vanishes in the limit when $k_{\text {eff }} r_{0} \ll 1$. Since $k_{\text {eff }} r_{0} \ll 1$, this approach gives rise to two independent equations that allow us to determine $\varepsilon_{\text {eff }}$ and $\mu_{\text {eff }}$ separately. In this approach, effective medium theory has been derived in the longwavelength limit, ${ }^{9-16}$ which is defined by the condition that $k_{0} r_{0} \ll 1,{ }^{11}$ where $k_{0}$ is the wave vector in the background material. In the long-wavelength limit, dipole approximation can be applied to both the background medium of the coated particle and the effective medium. However, no restriction is imposed on the magnitude of $k_{s} r_{s}$, where $k_{s}$ is the wave vector in the granular particle. The long-wavelength theory has been applied to various metamaterials. ${ }^{7,17}$ Recently, the effective medium properties have also been obtained numerically from the transmission and reflection amplitudes of a slab. $^{18}$

In this work, we show that it is possible to extend the long-wavelength effective medium theory, which is valid for $k_{0} r_{0} \ll 1$, to a region where $k_{0} r_{0}>1$. By doing so, our theory can be applied to a much larger frequency range than the standard long-wavelength theory. In order to test our theory, we have performed the band-structure calculations for a periodic composite containing complicated Mie resonances for each scatterer in two dimensions. The band-structure results give the exact dispersion relations within numerical error. We found that our theory is indeed capable of accurately reproducing almost all the main features found in the numerical calculations in a large frequency range that goes beyond the long-wavelength limit. The formulation of our theory is given in Sec. II. In Sec. III, we present the results of our band-structure calculations and compare the results with those obtained from our new effective medium theory. Conclusions are given in Sec. IV.

\section{EFFECTIVE MEDIUM THEORY: BEYOND THE LONG-WAVELENGTH LIMIT}

In this section, we give the formulation of our effective medium theory for both two and three dimensions. The system considered here is a composite consisting of a periodic array of cylinders (or spheres) possessing dielectric permittivity, $\varepsilon_{s}$, and magnetic permeability, $\mu_{s}$, with radii, $r_{s}$, embedded in a background medium with dielectric permittivity, $\varepsilon_{0}$, and magnetic permeability, $\mu_{0}$, in $2 \mathrm{D}(3 \mathrm{D})$. For simplic- 


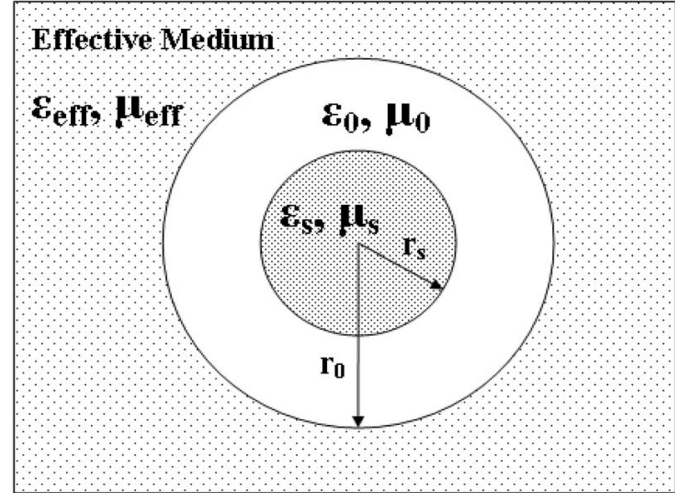

FIG. 1. Microstructure of the composite.

ity, we consider the background medium as a vacuum. In the cermet topology, the standard effective medium theory considers the scattering of an electromagnetic wave in an effective medium by a coated cylinder (or sphere) of radius $r_{o}$ with its area (or volume) chosen to be that of a unit cell as shown in Fig. 1. The inner cylinder (or sphere) of the coated cylinder (or sphere) represents the particle in the composite, whereas the outer layer represents the background medium. This coated cylinder (or sphere) represents the microstructure of the composite. The effective medium parameters, i.e., $\varepsilon_{\text {eff }}$ and $\mu_{\text {eff }}$, are determined by the condition that the total scattering vanishes in the limit of $k_{\text {eff }} r_{0} \ll 1{ }^{6,19}$

In a $2 \mathrm{D}$ system, we consider only the case of transverse magnetic (TM) waves, i.e., the electric field is parallel to the cylinder axis. Due to the symmetry of Maxwell's equations, the effective medium parameters for transverse electric (TE) waves can be obtained from that of TM waves by interchanging the roles of permittivity and permeability. For TM waves, the electric field is parallel to the cylinder and its solutions in the effective medium can be written as

$$
E_{z}(\vec{r})=\sum_{m} a_{m}(\mathrm{eff}) J_{m}\left(k_{\mathrm{eff}} r\right) e^{i m \theta}+b_{m}(\mathrm{eff}) H_{m}^{(1)}\left(k_{\mathrm{eff}} r\right) e^{i m \theta},
$$

where $k_{\text {eff }}=\omega \sqrt{\varepsilon_{\text {eff }}} \sqrt{\mu_{\text {eff }}}$ and $\omega$ is the frequency of the wave. Similarly, the electric field in the background medium of the coated cylinder takes the form

$$
E_{z}(\vec{r})=\sum_{m} a_{m}(0) J_{m}\left(k_{0} r\right) e^{i m \theta}+b_{m}(0) H_{m}^{(1)}\left(k_{0} r\right) e^{i m \theta},
$$

where $k_{0}=\omega \sqrt{\varepsilon_{0}} \sqrt{\mu_{0}}$. Here $J_{m}(x)$ and $H_{m}^{(1)}(x)$ are the Bessel function and Hankel function of the first kind, respectively. The total scattering cross section of the coated cylinder is given by

$$
C_{\text {sca }}=\frac{4}{k_{\text {eff }}} \sum_{m} \mid\left. D_{m}(\text { tot })\right|^{2},
$$

where $D_{m}($ tot $) \equiv b_{m}($ eff $) / a_{m}$ (eff) are the Mie scattering coefficients of the whole coated cylinder. In the case of $k_{\text {eff }} r_{0}$ $\ll 1, C_{\text {sca }}$ is dominated by $m=0$ and $m=1$ terms in Eq. (3). Thus, we can set the conditions for an effective medium as $D_{0}($ tot $)=0$ and $D_{1}$ (tot) $=0$, or, equivalently, $b_{0}($ eff $)=0$ and $b_{1}(\mathrm{eff})=0$. By matching the boundary conditions on the interface of the background and effective medium, we obtain

$$
\left(\begin{array}{l}
a_{m}(\text { eff }) \\
b_{m}(\text { eff })
\end{array}\right)=F\left(\begin{array}{ll}
A_{11} & A_{12} \\
A_{21} & A_{22}
\end{array}\right)\left(\begin{array}{l}
a_{m}(0) \\
b_{m}(0)
\end{array}\right)
$$

with $F=\left[k_{e} \mu_{0} J_{m}\left(k_{e} r_{0}\right) H_{m}^{(1)^{\prime}}\left(k_{e} r_{0}\right)-k_{e} \mu_{0} J_{m}^{\prime}\left(k_{e} r_{0}\right) H_{m}^{(1)}\left(k_{e} r_{0}\right)\right]^{-1}$ and

$$
A_{11}=k_{e} \mu_{0} J_{m}\left(k_{0} r_{0}\right) H_{m}^{(1)^{\prime}}\left(k_{e} r_{0}\right)-k_{0} \mu_{e} J_{m}^{\prime}\left(k_{0} r_{0}\right) H_{m}^{(1)}\left(k_{e} r_{0}\right)
$$

$$
\begin{gathered}
A_{12}=k_{e} \mu_{0} H_{m}^{(1)}\left(k_{0} r_{0}\right) H_{m}^{(1)^{\prime}}\left(k_{e} r_{0}\right)-k_{0} \mu_{e} H_{m}^{(1)^{\prime}}\left(k_{0} r_{0}\right) H_{m}^{(1)}\left(k_{e} r_{0}\right), \\
A_{21}=k_{0} \mu_{e} J_{m}\left(k_{e} r_{0}\right) J_{m}^{\prime}\left(k_{0} r_{0}\right)-k_{e} \mu_{0} J_{m}\left(k_{0} r_{0}\right) J_{m}^{\prime}\left(k_{e} r_{0}\right), \\
A_{22}=k_{0} \mu_{e} J_{m}\left(k_{e} r_{0}\right) H_{m}^{(1)^{\prime}}\left(k_{0} r_{0}\right)-k_{e} \mu_{0} H_{m}^{(1)}\left(k_{0} r_{0}\right) J_{m}^{\prime}\left(k_{e} r_{0}\right) .
\end{gathered}
$$

By using the conditions $b_{0}($ eff $)=0$ and $b_{1}($ eff $)=0$, we obtain from Eq. (4)

$$
\frac{A_{21}}{A_{22}}=-\frac{b_{m}(0)}{a_{m}(0)}=-D_{m}, \quad m=0,1,
$$

where $D_{m}$ represent the Mie scattering coefficients of the particle and have the form

$$
D_{m}=\frac{\mu_{0} k_{s} J_{m}^{\prime}\left(k_{s} r_{s}\right) J_{m}\left(k_{0} r_{s}\right)-\mu_{s} k_{0} J_{m}\left(k_{s} r_{s}\right) J_{m}^{\prime}\left(k_{0} r_{s}\right)}{\mu_{s} k_{0} J_{m}\left(k_{s} r_{s}\right) H_{m}^{(1)}\left(k_{0} r_{s}\right)-\mu_{0} k_{s} J_{m}^{\prime}\left(k_{s} r_{s}\right) H_{m}^{(1)}\left(k_{0} r_{s}\right)},
$$

where $r_{s}$ is the radius of the inner cylinder, and $\varepsilon_{s}$ and $\mu_{s}$ are, respectively, the permittivity and permeability of the scatterers and $k_{s}=\omega \sqrt{\varepsilon_{s}} \sqrt{\mu_{s}}$. Equation (5) is the basis of three different effective medium theories to be derived below depending on the level of approximation.

First, we consider the limit of $k_{e} r_{o} \ll 1$ without imposing any restriction on the magnitude of $k_{o} r_{o}, k_{o} r_{s}$, and $k_{s} r_{s}$. In this case, we can approximate the functions $J_{m}(x), J_{m}^{\prime}(x)$, $H_{m}^{(1)}(x)$, and $H_{m}^{(1)^{\prime}}(x)$ with $x \equiv k_{e} r_{o}$ in $A_{21}$ and $A_{22}$ by $J_{0}(x)$ $\cong 1, J_{1}(x) \cong x / 2, J_{0}^{\prime}(x) \cong-x / 2$, and $J_{1}^{\prime}(x) \cong \frac{1}{2}$, and $H_{0}^{(1)}(x)$ $\cong 1+i(2 / \pi) \ln x, H_{1}^{(1)}(x) \cong(x / 2)-i(2 / \pi x), H_{0}^{(1)^{\prime}}(x) \cong-(x / 2)$ $+i(2 / \pi x)$, and $H_{1}^{(1)^{\prime}}(x) \cong \frac{1}{2}+i\left(2 / \pi x^{2}\right)$. With these approximations, Eq. (5) reduces to

$$
\frac{\varepsilon_{\mathrm{eff}}(\omega)+2 \varepsilon_{0} \frac{J_{0}{ }^{\prime}\left(k_{o} r_{o}\right)}{k_{o} r_{o} J_{0}\left(k_{o} r_{o}\right)}}{\varepsilon_{\mathrm{eff}}(\omega)+2 \varepsilon_{0} \frac{Y_{0}^{\prime}\left(k_{o} r_{o}\right)}{k_{o} r_{o} Y_{0}\left(k_{o} r_{o}\right)}}=\frac{Y_{0}\left(k_{o} r_{o}\right)}{i J_{0}\left(k_{o} r_{o}\right)}\left(\frac{D_{0}(\omega)}{1+D_{0}(\omega)}\right)
$$




$$
\frac{\mu_{\mathrm{eff}}(\omega)-\mu_{0} \frac{J_{1}\left(k_{o} r_{o}\right)}{k_{o} r_{o} J_{1}^{\prime}\left(k_{o} r_{o}\right)}}{\mu_{\mathrm{eff}}(\omega)-\mu_{0} \frac{Y_{1}\left(k_{o} r_{o}\right)}{k_{o} r_{o} Y_{1}^{\prime}\left(k_{o} r_{o}\right)}}=\frac{Y_{1}^{\prime}\left(k_{o} r_{o}\right)}{i J_{1}^{\prime}\left(k_{o} r_{o}\right)}\left(\frac{D_{1}(\omega)}{1+D_{1}(\omega)}\right),
$$

where $Y_{m}(x)$ is the Neumamn function and $H_{m}^{(1)}(x)=J_{m}(x)$ $+i Y_{m}(x)$. Equations (7a) and (7b) are the $2 \mathrm{D}$ results of our effective medium theory for effective permittivity, $\varepsilon_{\text {eff }}(\omega)$, and permeability, $\mu_{\mathrm{eff}}(\omega)$. Since Eq. (7) does not require the parameters $k_{o} r_{o}, k_{o} r_{s}$, and $k_{s} r_{s}$ to be small, its region of validity can go beyond that of the long-wavelength limit, i.e., $k_{e} r_{o} \ll 1$ and $k_{o} r_{o} \ll 1 .{ }^{11}$ It should be pointed out that the factors $1+D_{m}$ in the denominators of Eqs. (7a) and (7b) are important to keep the sum rule correct. ${ }^{20}$ They compensate for the radiative loss arising from the real parts of $D_{0}$ and $D_{1}$ so that both $\varepsilon_{\text {eff }}(\omega)$ and $\mu_{\text {eff }}(\omega)$ become real if $\varepsilon_{s}(\omega), \varepsilon_{0}(\omega)$, $\mu_{s}(\omega)$, and $\mu_{0}(\omega)$ are all real. This can be seen if we apply the optical theorem, i.e., $\operatorname{Re}\left(D_{0}\right)=-\left|D_{0}\right|^{2}$ and $\operatorname{Re}\left(D_{1}\right)=$ $-\left|D_{1}\right|^{2}$ to the right-hand side of Eqs. (7a) and (7b). Since Eq. (7) maintains the energy conservation, it can be applied meaningfully to absorptive media. Furthermore, since Eq. (7) requires only a small value of $k_{e} r_{o} \equiv n_{\mathrm{eff}} k_{o} r_{o}$, it could still be valid at large frequencies as long as the effective refractive index, $n_{\text {eff }} \equiv \sqrt{\varepsilon_{\text {eff }} / \varepsilon_{0}} \sqrt{\mu_{\text {eff }} / \mu_{0}}$, is small.

We now consider the long-wavelength limit. In addition to $k_{e} r_{o} \ll 1$, we also require $k_{o} r_{o} \ll 1$. We use the same approximations described before for the functions $J_{m}(x), J_{m}^{\prime}(x)$, and $H_{m}^{(1)}(x)$ but with $x \equiv k_{o} r_{o}$ in Eq. (7). Equation (7) then reduces to

$$
\frac{\varepsilon_{\mathrm{eff}}-\varepsilon_{0}}{\varepsilon_{0}}=p \frac{\widetilde{\varepsilon}_{s}-\varepsilon_{0}}{\varepsilon_{0}} \quad \text { and } \quad \frac{\mu_{\mathrm{eff}}-\mu_{0}}{\mu_{\mathrm{eff}}+\mu_{0}}=p \frac{\tilde{\mu}_{s}-\mu_{0}}{\tilde{\mu}_{s}+\mu_{0}} \text {, }
$$

where $\widetilde{\varepsilon}_{s}$ and $\tilde{\mu}_{s}$ are, respectively, the renormalized permittivity and permeability of the cylinder with $\widetilde{\varepsilon}_{s}=2 F_{2}\left(k_{s} r_{s}\right) \varepsilon_{s}$, $\tilde{\mu}_{s}=\left\{F_{2}\left(k_{s} r_{s}\right) /\left[1-F_{2}\left(k_{s} r_{s}\right)\right]\right\} \mu_{s}, \quad$ and $\quad F_{2}(x)=J_{1}(x) /\left[x J_{0}(x)\right]$. Here, $p \equiv\left(r_{s} / r_{o}\right)^{2}$ represents the filling ratio of the cylinder. We note that Eq. (8) has been derived previously in a different form although the accuracy of this result has not yet been examined. ${ }^{13}$ Although Eq. (8) is simpler than Eq. (7), its region of validity is expected to be confined to a lower frequency region than that of Eq. (7). The quasistatic limit is reached if we further consider the condition of $k_{s} r_{s} \ll 1$ inside the scatterers. In this limit, $F_{2}\left(k_{s} r_{s}\right)$ approaches 0.5 . Thus, $\widetilde{\varepsilon}_{s}$ and $\tilde{\mu}_{s}$ reduce to their bare values, $\varepsilon_{s}$ and $\mu_{s}$, and Eq. (8) reduces to the Maxwell-Garnett (MG) theory in 2D, in which the electric and magnetic phenomena are decoupled. Since the derivations of Eqs. (7) and (8) do not require $k_{s} r_{s} \ll 1$, they should hold even when the cylinder possesses complicated resonant structures and when $k_{s} r_{s}$ is large.

For 3D systems, the scatterer becomes a sphere and the coated cylinder becomes a coated sphere. The electric field in the effective medium now has the form

$$
\vec{E}(\vec{r})=\sum_{l, m \sigma=E, H} a_{l m}^{(\sigma)}(\mathrm{eff}) \vec{J}_{l m}^{(\sigma)}\left(k_{\mathrm{eff}} \vec{r}\right)+b_{l m}^{(\sigma)}(\mathrm{eff}) \vec{H}_{l m}^{(\sigma)}\left(k_{\mathrm{eff}} \vec{r}\right),
$$

and in the background medium as

$$
\vec{E}(\vec{r})=\sum_{l, m \sigma=E, H} a_{l m}^{(\sigma)}(0) \vec{J}_{l m}^{(\sigma)}\left(k_{0} \vec{r}\right)+b_{l m}^{(\sigma)}(0) \vec{H}_{l m}^{(\sigma)}\left(k_{0} \vec{r}\right)
$$

Here, $\vec{J}_{l m}^{(\sigma)}$ and $\vec{H}_{l m}^{(\sigma)}$ are the vector spherical harmonics with $\sigma=E$ and $H$ denoting, respectively, the electric and magnetic multipoles. The scattering cross section of the coated particle is governed by

$$
C_{\mathrm{sca}}=\frac{2 \pi}{k_{\text {eff }}^{2}} \sum_{l=1}^{\infty}(2 l+1)\left[\mid\left. D_{l}^{(H)}(\text { tot })\right|^{2}+\mid\left. D_{l}^{(E)}(\text { tot })\right|^{2}\right] .
$$

In this case, $C_{\mathrm{sca}}$ is dominated by two $l=1$ terms. Therefore, the effective medium conditions are $D_{1}^{(E)}($ tot $)=0$ and $D_{1}^{(H)}($ tot $)=0$, which in turn imply that $b_{1 m}^{(E)}($ eff $)=0$ and $b_{1 m}^{(H)}(\mathrm{eff})=0$. By matching the boundary condition on the interface of the background and effective medium, we obtain

$$
\left(\begin{array}{l}
a_{1 m}^{(\sigma)}(\mathrm{eff}) \\
b_{1 m}^{(\sigma)}(\mathrm{eff})
\end{array}\right)=F\left(\begin{array}{ll}
A_{11}^{(\sigma)} & A_{12}^{(\sigma)} \\
A_{21}^{(\sigma)} & A_{22}^{(\sigma)}
\end{array}\right)\left(\begin{array}{l}
a_{1 m}^{(\sigma)}(0) \\
b_{1 m}^{(\sigma)}(0)
\end{array}\right),
$$

with $F=\left(k_{0} \mu_{0}\left[\psi_{1}\left(k_{\text {eff }} r_{0}\right) \xi_{1}^{\prime}\left(k_{\text {eff }} r_{0}\right)-\xi_{1}\left(k_{\text {eff }} r_{0}\right) \psi_{1}^{\prime}\left(k_{\text {eff }} r_{0}\right)\right]\right)^{-1}$ and

$$
\begin{aligned}
& A_{11}^{(\sigma)}=\alpha^{(\sigma)} \psi_{1}\left(k_{0} r_{0}\right) \xi_{1}{ }^{\prime}\left(k_{\mathrm{eff}} r_{0}\right)-\beta^{(\sigma)} \xi_{1}\left(k_{\mathrm{eff}} r_{0}\right) \psi_{1}{ }^{\prime}\left(k_{0} r_{0}\right), \\
& A_{12}^{(\sigma)}=\alpha^{(\sigma)} \xi_{1}\left(k_{0} r_{0}\right) \xi_{1}{ }^{\prime}\left(k_{\mathrm{eff}} r_{0}\right)-\beta^{(\sigma)} \xi_{1}\left(k_{\mathrm{eff}} r_{0}\right) \xi_{1}{ }^{\prime}\left(k_{0} r_{0}\right), \\
& A_{21}^{(\sigma)}=\beta^{(\sigma)} \psi_{1}\left(k_{\mathrm{eff}} r_{0}\right) \psi_{1}{ }^{\prime}\left(k_{0} r_{0}\right)-\alpha^{(\sigma)} \psi_{1}\left(k_{0} r_{0}\right) \psi_{1}{ }^{\prime}\left(k_{\mathrm{eff}} r_{0}\right), \\
& A_{22}^{(\sigma)}=\beta^{(\sigma)} \psi_{1}\left(k_{\mathrm{eff}} r_{0}\right) \xi_{1}{ }^{\prime}\left(k_{0} r_{0}\right)-\alpha^{(\sigma)} \xi_{1}\left(k_{0} r_{0}\right) \psi_{1}{ }^{\prime}\left(k_{\mathrm{eff}} r_{0}\right),
\end{aligned}
$$

where $\alpha^{(E)}=k_{0} \mu_{\mathrm{eff}}, \beta^{(E)}=k_{\mathrm{eff}} \mu_{0}, \alpha^{(H)}=\beta^{(E)}, \beta^{(H)}=\alpha^{(E)}, \psi_{l}(x)$ $=x j_{l}(x)$, and $\xi_{l}(x)=x h_{l}^{(1)}(x)$. The functions $j_{l}(x)$ and $h_{l}^{(1)}(x)$ are, respectively, the spherical Bessel and Hankel functions. The conditions of $b_{1 m}^{(E)}(\mathrm{eff})=0$ and $b_{1 m}^{(H)}(\mathrm{eff})=0$ give

$$
\frac{A_{21}^{(\sigma)}}{A_{22}^{(\sigma)}}=-\frac{b_{1 m}^{(\sigma)}(0)}{a_{1 m}^{(\sigma)}(0)}=-D_{1}^{(\sigma)},
$$

where $D_{1}^{(\sigma)}$ represent the Mie scattering coefficients of the particle and have the forms

$$
\begin{aligned}
& D_{1}^{(E)}=\frac{-k_{s} \mu_{0} \psi_{1}\left(k_{s} r_{s}\right) \psi_{1}^{\prime}\left(k_{0} r_{s}\right)+k_{0} \mu_{s} \psi_{1}\left(k_{0} r_{s}\right) \psi_{1}{ }^{\prime}\left(k_{s} r_{s}\right)}{k_{s} \mu_{0} \psi_{1}\left(k_{s} r_{s}\right) \xi_{1}{ }^{\prime}\left(k_{0} r_{s}\right)-k_{0} \mu_{s} \xi_{1}\left(k_{0} r_{s}\right) \psi_{1}^{\prime}\left(k_{s} r_{s}\right)} \\
& D_{1}^{(H)}=\frac{-k_{0} \mu_{s} \psi_{1}\left(k_{s} r_{s}\right) \psi_{1}^{\prime}\left(k_{0} r_{s}\right)+k_{s} \mu_{0} \psi_{1}\left(k_{0} r_{s}\right) \psi_{1}^{\prime}\left(k_{s} r_{s}\right)}{k_{0} \mu_{s} \psi_{1}\left(k_{s} r_{s}\right) \xi_{1}^{\prime}\left(k_{0} r_{s}\right)-k_{s} \mu_{0} \xi_{1}\left(k_{0} r_{s}\right) \psi_{1}^{\prime}\left(k_{s} r_{s}\right)},
\end{aligned}
$$

where $r_{s}$ is the radius of the inner sphere, $\varepsilon_{s}$ and $\mu_{s}$ are, respectively, the permittivity and permeability of the scatterers, and $k_{s}=\omega \sqrt{\varepsilon_{s}} \sqrt{\mu_{s}}$. In the limit of $k_{\text {eff }} r_{0} \ll 1$, we can approximate the functions $j_{1}\left(k_{\mathrm{eff}} r_{0}\right)$ and $h_{1}\left(k_{\mathrm{eff}} r_{0}\right)$ in $A_{21}^{(\sigma)}$ and $A_{22}^{(\sigma)}$ in Eq. (13) by using $j_{1}(x) \cong x / 3$ and $h_{1}(x) \cong x / 3-i / x^{2}$. 
With these approximations, Eq. (13) gives the following two equations for $\varepsilon_{\mathrm{eff}}(\omega)$ and $\mu_{\mathrm{eff}}(\omega)$ :

$$
\begin{aligned}
& \varepsilon_{\mathrm{eff}}(\omega)-2 \varepsilon_{0} \frac{j_{1}\left(k_{o} r_{o}\right)}{j_{1}\left(k_{0} r_{0}\right)+k_{o} r_{o} j_{1}^{\prime}\left(k_{o} r_{o}\right)} \\
& \varepsilon_{\text {eff }}(\omega)-2 \varepsilon_{0} \frac{y_{1}\left(k_{o} r_{o}\right)}{y_{1}\left(k_{0} r_{0}\right)+k_{o} r_{o} y_{1}^{\prime}\left(k_{o} r_{o}\right)} \\
& =\frac{\left[y_{1}\left(k_{0} r_{0}\right)+k_{o} r_{o} y_{1}^{\prime}\left(k_{o} r_{o}\right)\right]}{i\left[j_{1}\left(k_{0} r_{0}\right)+k_{o} r_{o} j_{1}^{\prime}\left(k_{o} r_{o}\right)\right]} \frac{D_{1}^{(E)}(\omega)}{1+D_{1}^{(E)}(\omega)}, \\
& \mu_{\text {eff }}(\omega)-2 \mu_{0} \frac{j_{1}\left(k_{o} r_{o}\right)}{j_{1}\left(k_{0} r_{0}\right)+k_{o} r_{o} j_{1}^{\prime}\left(k_{o} r_{o}\right)} \\
& \mu_{\mathrm{eff}}(\omega)-2 \mu_{0} \frac{y_{1}\left(k_{o} r_{o}\right)}{y_{1}\left(k_{0} r_{0}\right)+k_{o} r_{o} y_{1}^{\prime}\left(k_{o} r_{o}\right)} \\
& =\frac{\left[y_{1}\left(k_{0} r_{0}\right)+k_{o} r_{o} y_{1}^{\prime}\left(k_{o} r_{o}\right)\right]}{i\left[j_{1}\left(k_{0} r_{0}\right)+k_{o} r_{o} j_{1}^{\prime}\left(k_{o} r_{o}\right)\right]} \frac{D_{1}^{(H)}(\omega)}{1+D_{1}^{(H)}(\omega)},
\end{aligned}
$$

where $y_{1}(x)$ is the spherical Neumann function and $h_{1}^{(1)}(x)$ $=j_{1}(x)+i y_{1}(x)$. Equation (15) is the $3 \mathrm{D}$ version of our effective medium theory. Since it does not require either $k_{s} r_{0} \ll 1$ or $k_{0} r_{0} \ll 1$, its region of validity can go beyond that of the long-wavelength theory obtained previously for 3D systems. Here, we show that the long-wavelength theory can be recovered if we further consider the condition of $k_{0} r_{0} \gg 1$ in Eq. (15). In this case, we can use the approximations $j_{1}(x)$ $\cong x / 3, h_{1}(x) \cong x / 3-i / x^{2}$, and $y_{1}(x) \cong-1 / x^{2}$ with $x=k_{0} r_{0}$ in Eq. (15) to obtain

$$
\begin{aligned}
& \frac{\varepsilon_{\mathrm{eff}}-\varepsilon_{0}}{\varepsilon_{\mathrm{eff}}+2 \varepsilon_{0}}=p \frac{\widetilde{\varepsilon}_{s}-\varepsilon_{0}}{\widetilde{\varepsilon}_{s}+2 \varepsilon_{0}}, \\
& \frac{\mu_{\mathrm{eff}}-\mu_{0}}{\mu_{\mathrm{eff}}+2 \mu_{0}}=p \frac{\widetilde{\mu}_{s}-\mu_{0}}{\tilde{\mu}_{s}+2 \mu_{0}},
\end{aligned}
$$

where

$$
\begin{aligned}
& \widetilde{\varepsilon}_{s}=\frac{2 F_{3}\left(k_{s} r_{s}\right)}{1-F_{3}\left(k_{s} r_{s}\right)} \varepsilon_{s}, \\
& \tilde{\mu}_{s}=\frac{2 F_{3}\left(k_{s} r_{s}\right)}{1-F_{3}\left(k_{s} r_{s}\right)} \mu_{s},
\end{aligned}
$$

with

$$
F_{3}(x)=\frac{1}{x^{2}}-\frac{\cot (x)}{x} .
$$

Here $p=\left(r_{s} / r_{0}\right)^{3}$ represents the filling ratio of the spherical particle. Equation (16) has been obtained previously by using different methods. ${ }^{9} 10,14$ In the quasistatic limit, i.e., $k_{s} r_{s} \ll 1$ inside the scatterers, $F_{3}\left(k_{s} r_{s}\right)$ approaches $1 / 3$. Again, $\widetilde{\varepsilon}_{s}$ and $\tilde{\mu}_{s}$ reduce to their bare values, $\varepsilon_{s}$ and $\mu_{s}$, and Eq. (16) reduces to the MG theory in $3 \mathrm{D} \cdot{ }^{19,21}$

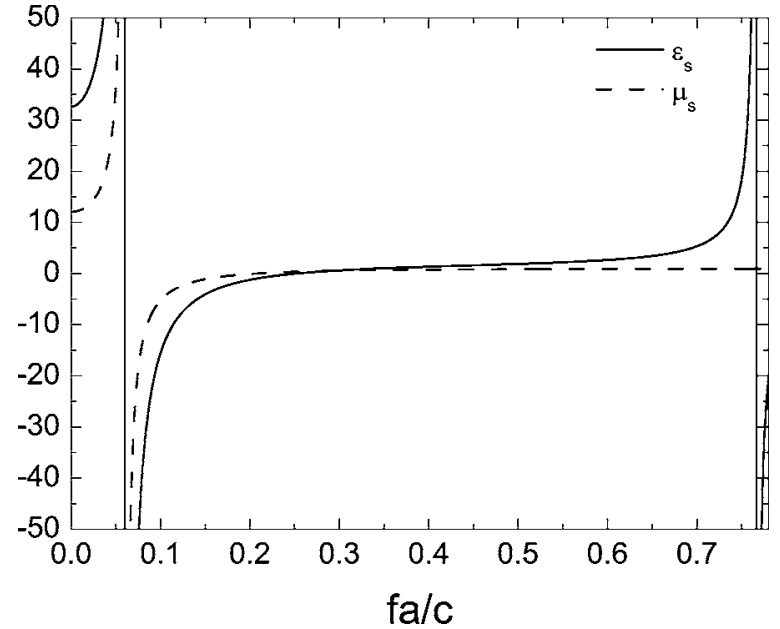

FIG. 2. Permittivity $\varepsilon_{s}$ and permeability $\mu_{s}$ of the scatterer.

\section{COMPARISON BETWEEN THE THEORY AND BAND-STRUCTURE CALCULATIONS IN TWO DIMENSIONS}

In order to verify the validity of Eq. (7), we performed band-structure calculations near the $\Gamma$ point for a periodic composite in two dimensions. The system we considered is a square array of cylindrical scatterers with a lattice constant, $a$, embedded in the air background. We assume that each scatterer has a radius $r_{s}=0.2 a$ and the following forms of dispersive permittivity and permeability:

$$
\begin{gathered}
\frac{\varepsilon_{s}(f a / c)}{\varepsilon_{0}}=1+\frac{\alpha_{1}}{\tilde{f}_{r 1}^{2}-(f a / c)^{2}}+\frac{\alpha_{2}}{\tilde{f}_{r 2}^{2}-(f a / c)^{2}}, \\
\frac{\mu_{s}(f a / c)}{\mu_{0}}=1+\frac{\alpha_{3}}{\widetilde{f}_{r 1}^{2}-(f a / c)^{2}},
\end{gathered}
$$

where $\alpha_{1}=0.11, \alpha_{2}=0.449, \alpha_{3}=0.04, \tilde{f}_{r 1}=0.06, \tilde{f}_{r 2}=0.767$, and $\widetilde{f} \equiv f a / c$ is a dimensionless frequency. Equation (17) is plotted in Fig. 2. It reduces to Eq. (13) of Ref. 22 when $a$ $=2 \mathrm{~cm}$. The band structures along the $\Gamma-X$ direction obtained from the multiple-scattering method ${ }^{23}$ are shown in Fig. 3. Figure 3 shows five major propagating bands in the following frequency regions: (i) $0<\tilde{f}<0.044$, (ii) $0.127<\tilde{f}$ $<0.144$, (iii) $0.161<\tilde{f}<0.465$, (iv) $0.505<\tilde{f}<0.689$, and (v) $0.717<\tilde{f}<0.734$. Besides these bands, there also exist three regions of densely packed flat bands. Two of them are located at $0.044<\tilde{f}<0.08$ and $0.747<\tilde{f}<\tilde{f}_{r 2}$. The presence of these two densely packed bands is due to the divergence of $\varepsilon_{s}$ at frequencies $\widetilde{f}_{r 1}$ and $\widetilde{f}_{r 2}$ and the divergence of $\mu_{s}$ at $\tilde{f}_{r 1}$ as shown in Eq. (17) or Fig. 2. Near these two frequencies, the Mie resonances become sharp and packed together, forming densely packed flat bands. Another region of flat bands is located near $\tilde{f}=0.1536$. The presence of flat bands in this region is due to the formation of "magnetic surface plasmons" in each cylinder when $\mu_{s}(0.1536) \cong-1$. The weak coupling of the surface plasmons between two nearestneighbors cylinders at various angular quantum numbers 


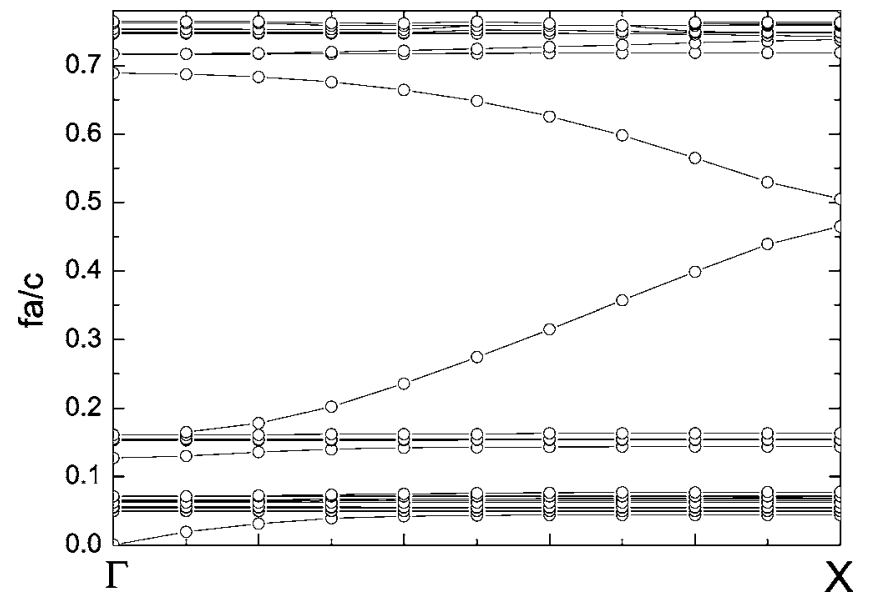

FIG. 3. Band structure along the $\Gamma-X$ direction calculated by the multiple-scattering method.

$(m \geqslant 2)$ forms closely packed flat bands. In Fig. 4(a), we replot the first three major propagating bands, i.e., (i) to (iii), with circles. Here, we are only interested in a region near the $\Gamma$ point, i.e., $0<K<\pi / 2 a$, so that a comparison between the band-structure calculations and effective medium theory is meaningful. If we replace $K$ by $k_{\text {eff }}$, the above range for $K$ translates to $0<k_{\text {eff }} r_{0}<0.89$. Beyond this range, it is clear that both Eqs. (7) and (8) become invalid. It should also be pointed out that within the above range of $K$, the band structure is rather isotropic around the $\Gamma$ point. This also makes an effective medium description of the system meaningful. In Figs. 4(b) and 4(c), we plot the functions of $\varepsilon_{\text {eff }}(\tilde{f})$ and $\mu_{\text {eff }}(\widetilde{f})$ obtained from Eqs. (7a) and (7b), respectively. From this, we can calculate the effective index of refraction, $n_{\text {eff }}$ $=\sqrt{\varepsilon_{\text {eff }}} / \varepsilon_{0} \sqrt{\mu_{\text {eff }} / \mu_{0}}$, and construct a dispersion relation through the relation $k_{\text {eff }}=2 \pi n_{\text {eff }} \tilde{f} / a$. The results are shown as solid curves in Fig. 4(a). It is clearly seen that Eq. (7) is capable of reproducing very accurately the band structures near the $\Gamma$ point.

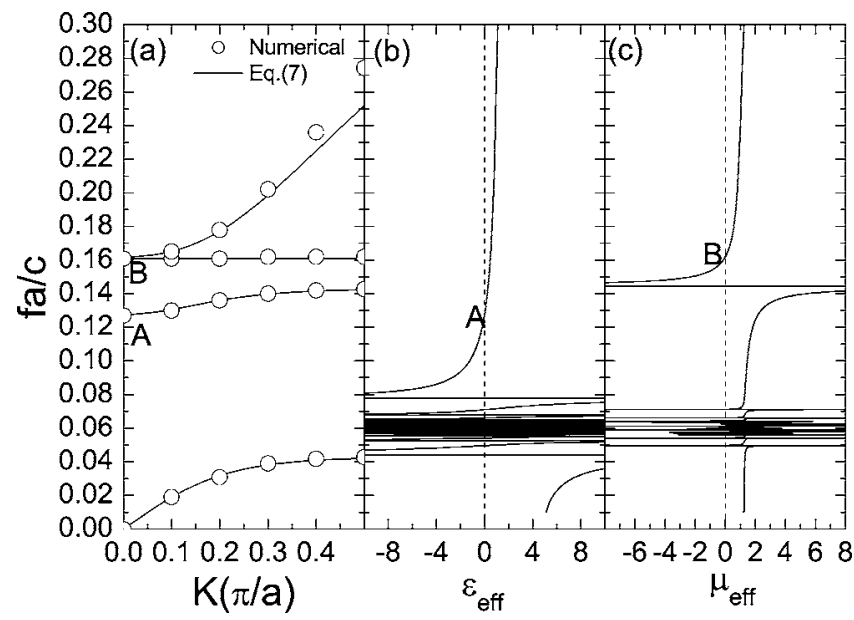

FIG. 4. (a) The circles denote the band structures near the $\Gamma$ point in the frequency range $0 \leqslant \tilde{f} \leqslant 0.3$. The solid curves represent the effective medium results of Eq. (7). (b) and (c) The effective permittivity and effective permeability obtained from Eq. (7), respectively.
Below, we draw a closer correspondence between the structures of $\varepsilon_{\text {eff }}(\widetilde{f})$ and $\mu_{\text {eff }}(\tilde{f})$ shown in Figs. 4(b) and 4(c) and the band structures shown in Fig. 4(a). For the lowest propagating band, i.e., $0<\tilde{f}<0.044$, both $\varepsilon_{\text {eff }}(\tilde{f})$ and $\mu_{\text {eff }}(\tilde{f})$ are positive and are increasing functions of frequency. Each of them diverges at a particular frequency near $\widetilde{f}=0.05$, where the first Mie resonance occurs for the case of $m=0$ and 1 , respectively. Due to the divergence in both $\varepsilon_{s}(\widetilde{f})$ and $\mu_{s}(\widetilde{f})$ in Eq. (17) at $\tilde{f}_{r 1}=0.06$, there exist infinite numbers of densely packed Mie resonances of both electric and magnetic natures in the frequency range $0.0442<\tilde{f}<0.0776$. We show later in detail that the resonances that appear in this range can provide a quantitative description of the densely packed flat bands shown in Fig. 3. For the frequencies between 0.0776 and 0.1278 , we have a region in which $\varepsilon_{\text {eff }}(\widetilde{f})<0$, but $\mu_{\text {eff }}(\tilde{f}) \geqslant 0$. This implies the presence of a band gap. Such a gap is indeed found in Fig. 3 or Fig. 4(a). As $\tilde{f}$ increases beyond $0.1278, \varepsilon_{\text {eff }}(\widetilde{f})$ becomes positive again, implying the existence of another propagating band. This band ends when $\mu_{\text {eff }}(\widetilde{f})$ turns negative at $\tilde{f}=0.1444$. This second band coincides with the second major band shown in Fig. 4(a), where the symbol "A" marks the beginning of this band at the $\Gamma$ point. When $\mu_{\text {eff }}(\tilde{f})$ becomes negative at $\tilde{f}=0.1444$, the second gap appears. This gap ends at $\tilde{f}=0.1618$ when $\mu_{\text {eff }}(\tilde{f})$ changes to positive again, above which we expect the existence of a third propagating band. The corresponding band is marked with the symbol "B" at the $\Gamma$ point in Fig. 4. It is interesting to point out that at point $\mathrm{B}$ there also exists a flat band. This flat band can be interpreted as a longitudinal mode that appears when $\mu_{\text {eff }}=0$ for $m=1$ resonances. This longitudinal mode does not exist for $m=0$ resonances and, therefore, we do not see a flat band at point $\mathrm{A}$, where $\varepsilon_{\text {eff }}$ $=0$. Thus, the band structures shown in Fig. 4(a) can be quantitatively reproduced by Eq. (7). Here, we should mention again that several closely packed flat bands near $\tilde{f}$ $=0.1536$ shown in Fig. 3 are not included in Fig. 4(a). These flat bands arise from "magnetic surface plasmons" with angular quantum numbers greater than or equal to 2 , and thus cannot be predicted by Eq. (7). We also calculated $\varepsilon_{\text {eff }}(\widetilde{f})$ and $\mu_{\text {eff }}(\widetilde{f})$ by using Eq. (8) and found almost the same results as that shown in Figs. 4(b) and 4(c). In fact, this is true as long as $\tilde{f}<0.3$, which is equivalent to $k_{0} r_{0}<1$ or $\lambda_{0} \equiv 2 \pi / k_{0}$ $>3.5 a$, where $\lambda_{0}$ is the wavelength in the background medium. This is expected as Eq. (8) is obtained from Eq. (7) by assuming a small value of $k_{0} r_{0}$. We show later that, when $\tilde{f}$ $>0.3$ (or equivalently, $k_{0} r_{0}>1$ ), the difference between Eqs. (7) and (8) becomes significant and Eq. (8) becomes invalid.

In Fig. 3, we find a large number of densely packed bands in the frequency range $0.0442<\tilde{f}<0.0776$. The presence of these bands is due to the occurrence of very sharp Mie resonances when $\tilde{f}$ is close to the first resonant frequency at $\tilde{f}_{r 1}$ $=0.06$. We show below that those bands are associated with $m=0$ or 1 states and can be accurately reproduced by the resonant structures of $\varepsilon_{\text {eff }}(\widetilde{f})$ and $\mu_{\text {eff }}(\widetilde{f})$ shown in Figs. 4(b) 


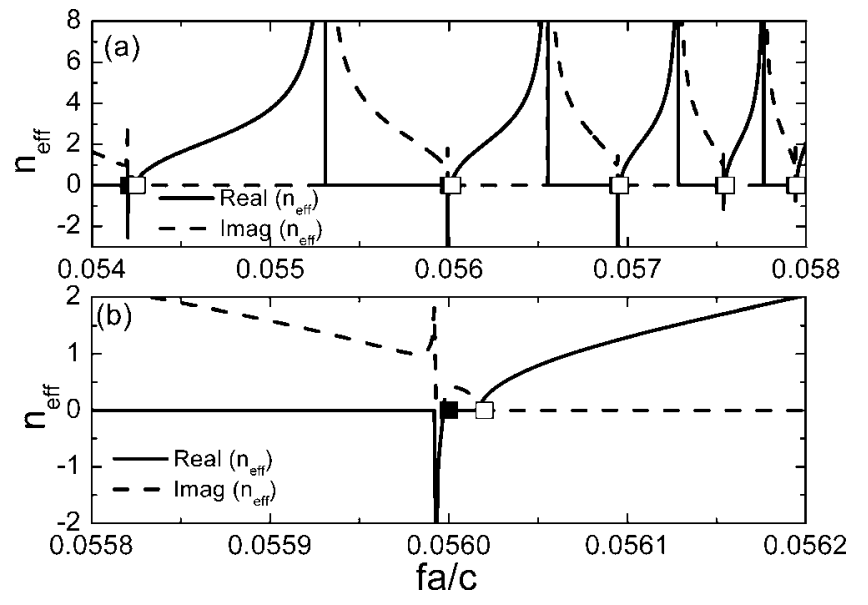

FIG. 5. Real part (solid curve) and imaginary part (dashed curve) of the effective refractive index, $n_{\text {eff }}$, obtained from Eq. (7). Squares denote the states at the $\Gamma$ point obtained from the bandstructure calculations. The solid squares correspond to the $m=0$ modes and the open squares correspond to the $m=1$ modes.

and 4(c). To illustrate this, in Fig. 5(a), we plot the real and imaginary parts of $n_{\text {eff }}(\widetilde{f})=\sqrt{\varepsilon_{\text {eff }}(\widetilde{f}) / \varepsilon_{0}} \sqrt{\mu_{\text {eff }}(\widetilde{f}) / \mu_{0}}$ in a small frequency range below $\tilde{f}_{r 1}: 0.054<\tilde{f}<0.058$. It should be mentioned that since both $\varepsilon_{\mathrm{eff}}(\tilde{f})$ and $\mu_{\mathrm{eff}}(\widetilde{f})$ are real functions of $\tilde{f}, n_{\text {eff }}(\tilde{f})$ can be either real or imaginary. The portion where $n_{\text {eff }}(\widetilde{f})$ is real indicates the existence of a propagating band, whereas the portion where $n_{\text {eff }}(\widetilde{f})$ is imaginary indicates a gap. The frequencies at which $n_{\text {eff }}(\tilde{f})=0$ predict the locations of the states at the $\Gamma$ point. Thus, Fig. 5(a) predicts the existence of at least four propagating bands starting from the $\Gamma$ point at $\tilde{f}=0.05425,0.05602,0.05696$, and 0.05755 . These points correspond to the case of $\mu_{\text {eff }}(\widetilde{f})=0$ caused by the $m=1$ resonance. Similar to the case of point B shown in Fig. 4(a), for each propagating band, there should also exist a flat band due to the appearance of a longitudinal mode when $\mu_{\text {eff }}(\widetilde{f})=0$. With a closer look to the left of these points, we find another set of frequencies at which $\varepsilon_{\text {eff }}(\tilde{f})=0$ due to the $m=0$ resonance. These frequencies are located at $\tilde{f}$ $=0.05421,0.05600,0.05695$, and 0.05754. The structures of the real and imaginary parts of $n_{\text {eff }}(\tilde{f})$ for a pair of resonances near $\tilde{f}=0.05600$ are magnified and shown in Fig. 5(b). From Fig. 5(b), we can see more clearly that each of these points corresponds to the end point of another propagating band, which is very narrow and has a negative refractive index. In Fig. 6(a), we plot the dispersion relations arising from this pair of resonances. The solid curves in Fig. 6(a) represent the results constructed from $n_{\text {eff }}(\widetilde{f})$, whereas the circles denote the results of the band-structure calculations. It should be pointed out that the lower flat band obtained by the bandstructure calculations is slightly bending downward. This is consistent with the negative refractive index predicted by Eq. (7) for the $m=0$ resonance. We also plot in Figs. 6(b) and $6(\mathrm{c})$ the functions $\varepsilon_{\mathrm{eff}}(\widetilde{f})$ and $\mu_{\mathrm{eff}}(\widetilde{f})$ obtained from Eq. (7). The zeros of these functions at $\tilde{f}=0.05600$ and 0.05602 pre-

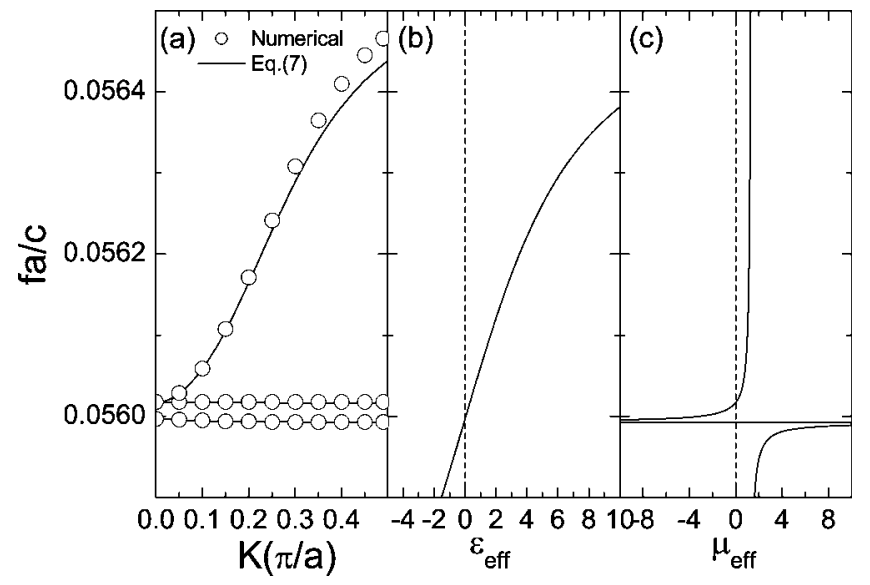

FIG. 6. (a) The circles denote the band structures near the $\Gamma$ point in the frequency range $0.0559 \leqslant \tilde{f} \leqslant 0.0565$. The solid curves represent the effective medium results of Eq. (7). (b) and (c) The effective permittivity and effective permeability obtained from Eq. (7), respectively.

dict accurately the locations of three bands obtained from the band-structure calculations. The excellent agreement shown in Fig. 6(a) actually holds for all other pairs of resonances we have investigated in this frequency range. To show this, we mark the states at the $\Gamma$ point obtained from band-structure calculations with solid squares for the $m=0$ states and open squares for the $m=1$ states in Fig. 5. This demonstrates again the validity as well as the accuracy of Eq. (7). Figures 5 and 6 show the behavior of densely packed bands for frequencies below $\tilde{f}_{r 1}$, where both $\varepsilon_{s}$ and $\mu_{s}$ are positive. For frequencies above $\tilde{f}_{r 1}$, both $\varepsilon_{s}$ and $\mu_{s}$ become negative as shown in Fig. 2 . In this case, the scatterer has a negative refractive index. The behavior of densely packed bands in this frequency region is slightly different from that shown in Figs. 5 and 6. In Fig. 7(a), we plot the real and imaginary parts of $n_{\text {eff }}(\widetilde{f})$ in a small frequency range $0.062<\tilde{f}<0.072$. A particular pair of resonances is magnified in Fig. 7(b). The results of bandstructure calculations at the $\Gamma$ point are shown with solid squares for the $m=0$ states and open squares for the $m=1$ states. The accuracy of Eq. (7) is again demonstrated. However, compared to Fig. 5(b), we find that the $m=0$ state shown in Fig. 7(b) corresponds to the starting point of a very narrow propagating band with a positive refractive index. Indeed, our band-structure calculations show that this flat band is slightly bending upward.

We have mentioned before that Eq. (8) produces almost the same results as that of Eq. (7) in the long-wavelength limit when $\tilde{f}<0.3$. Below, we discuss the region beyond the long-wavelength limit, i.e., $\tilde{f}>0.3$ (or equivalently, $k_{0} r_{0}>1$ ). In Figs. 8(a) and 8(d), we compare the dispersion relations obtained from Eqs. (7) and (8), respectively (solid curves) with those from our band-structure calculations (open circles) for the last two major propagating bands shown in Fig. 3, i.e., (iv) $0.505<\tilde{f}<0.689$ and (v) $0.717<\tilde{f}<0.734$ for $0<K<\pi / 2 a$. Also shown in Figs. 8(b) and 8(c) are the functions $\varepsilon_{\mathrm{eff}}(\tilde{f})$ and $\mu_{\mathrm{eff}}(\tilde{f})$ obtained from Eq. (7). The cor- 

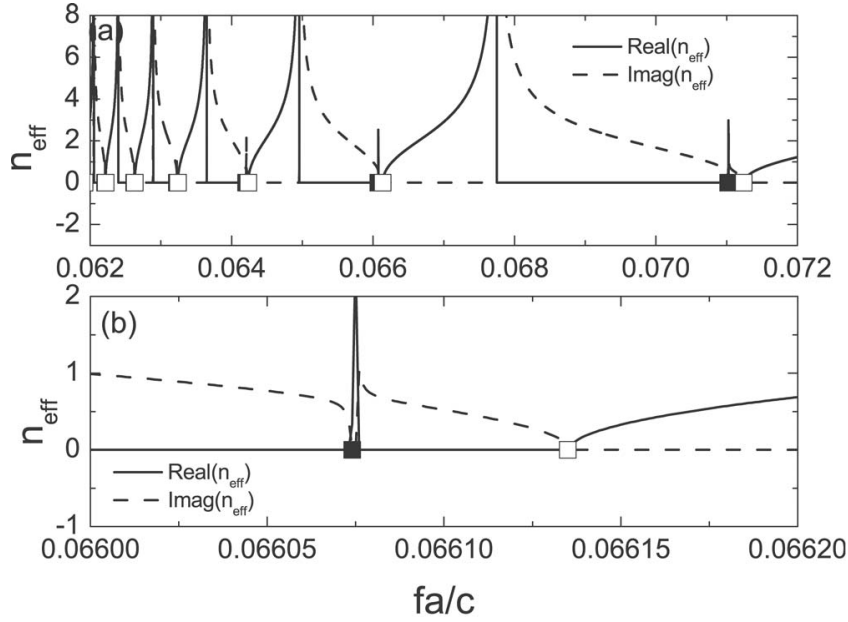

FIG. 7. Real part (solid curve) and imaginary part (dashed curve) of the effective refractive index, $n_{\text {eff }}$, obtained from Eq. (7). Squares denote the states at the $\Gamma$ point obtained from the bandstructure calculations. The solid squares correspond to the $m=0$ modes and the open squares correspond to the $m=1$ modes.

responding results of Eq. (8) are shown in Figs. 8(e) and 8(f). It is quite clear that Eqs. (7) and (8) predict very different effective medium behaviors in this frequency range. It is also clear from Fig. 8(a) that Eq. (7) is capable of reproducing the correct band structures. However, Eq. (8) does not predict any bands in the region shown in Fig. 8(d), and, therefore, fails completely in this case. It is interesting to point out that, for $\tilde{f}<0.694$, both $\varepsilon_{\text {eff }}(\widetilde{f})$ and $\mu_{\text {eff }}(\widetilde{f})$ are negative as shown in Figs. 8(b) and 8(c), which predicts a propagating band with a negative refractive index. This band ends at the $\Gamma$ point when $\varepsilon_{\text {eff }}(\tilde{f}=0.6940)=0$. The band-structure calculations denoted by circles in Fig. 8(a) indeed show the existence of this band with a negative curvature. ${ }^{24}$ However, Eq.

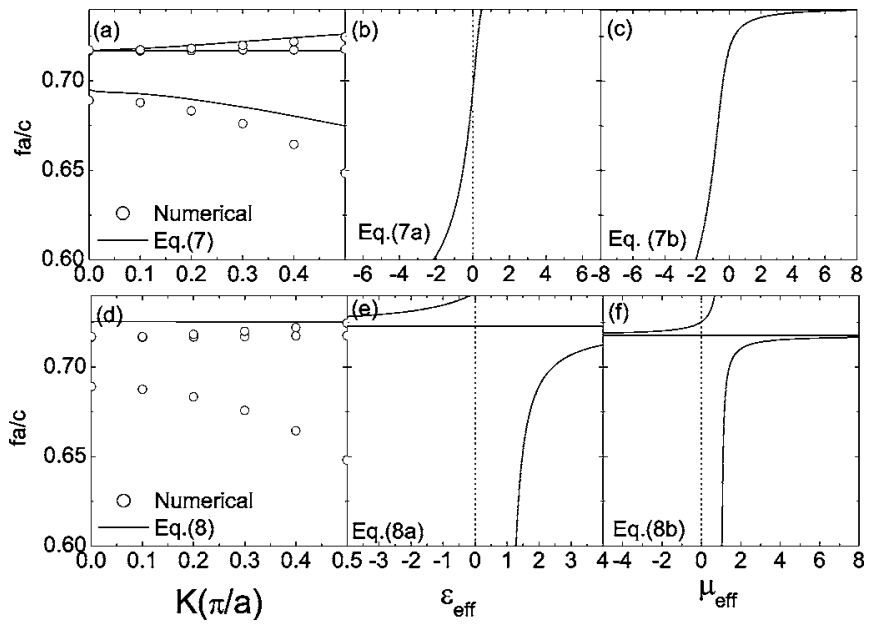

FIG. 8. (a) The circles denote the band structures near the $\Gamma$ point in the frequency range $0.6 \leqslant \tilde{f} \leqslant 0.75$. The solid curves represent the effective medium results of Eq. (7). (b) and (c) The effective permittivity and effective permeability obtained from Eq. (7), respectively. (d) and (e) show the same variables as (a)-(c) but all solid curves are obtained from Eq. (8).
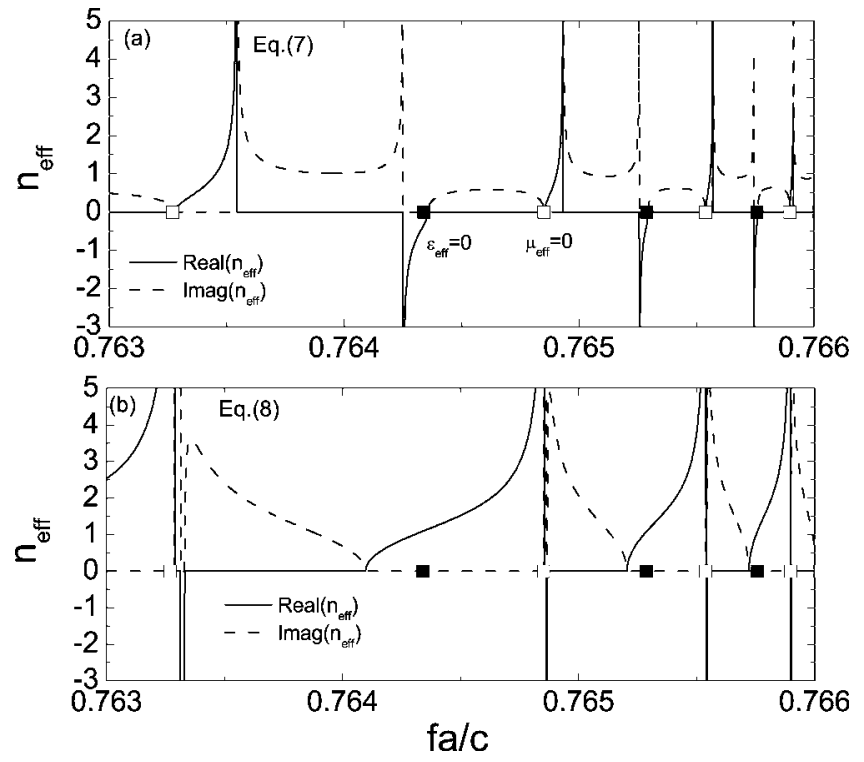

FIG. 9. Real part (solid curve) and imaginary part (dashed curve) of the effective refractive index, $n_{\text {eff }}$, obtained, respectively, from (a) Eq. (7) and (b) Eq. (8). Squares denote the states at the $\Gamma$ point obtained from the band-structure calculations. The solid squares correspond to the $m=0$ modes and the open squares correspond to $m=1$ modes, respectively.

(8) does not predict the existence of this negative- $n$ band. In fact, Figs. 8(e) and 8(f) show that the functions $\varepsilon_{\text {eff }}(\tilde{f})$ and $\mu_{\text {eff }}(\widetilde{f})$ are both positive. From Figs. 8(b) and 8(c), we also see a frequency region between 0.689 and 0.717 , in which $\varepsilon_{\text {eff }}(\widetilde{f})>0$ but $\mu_{\text {eff }}(\widetilde{f})<0$, indicating a gap. When $\tilde{f}>0.717$, both $\varepsilon_{\text {eff }}(\widetilde{f})$ and $\mu_{\text {eff }}(\widetilde{f})$ become positive again, giving rise to another propagating band but with a positive refractive index. Since this band starts at the $\Gamma$ point and is caused by the $m=1$ resonance, there also exists a flat band at $\tilde{f}=0.717$ due to the longitudinal mode. All these predictions of Eq. (7) are confirmed by the band-structure calculations shown in Fig. $8(a)$. In particular, the state at the $\Gamma$ point coincides with that predicted by Eq. (7). On the contrary, Eq. (8) predicts a negative- $n$ band ending at $\tilde{f}=0.725$ at the $\Gamma$ point, as can be seen from Figs. 8(e) and 8(f). This shows again that Eq. (8) is entirely inapplicable in this frequency region.

As mentioned before, due to the divergence of $\varepsilon_{s}$ in Eq. (17) at $\widetilde{f}_{r 2}=0.767$, there exists another region of closely packed flat bands located at $0.747<\tilde{f}<\tilde{f}_{r 2}$, induced by the Mie resonances. In order to compare the theories with bandstructure calculations in detail, we focus our discussions on a small frequency range, i.e., $0.763<\tilde{f}<0.766$, which includes seven resonances. In Fig. 9(a), we plot the real and imaginary parts of $n_{\text {eff }}(\tilde{f})$ obtained from Eq. (7). The behaviors of $\varepsilon_{\text {eff }}(\widetilde{f})$ and $\mu_{\text {eff }}(\widetilde{f})$ near a pair of zeros at $\widetilde{f}=0.76436$ and 0.76485 are magnified and shown in Figs. 10(b) and 10(c). The corresponding results obtained from Eq. (8) are also plotted in Fig. 9(b) for $n_{\text {eff }}(\tilde{f})$ and Figs. 10(e) and 10(f) for $\varepsilon_{\text {eff }}(\widetilde{f})$ and $\mu_{\text {eff }}(\widetilde{f})$, respectively. It is easy to see that Figs. 9(a) and 9(b) exhibit very different structures for both the 

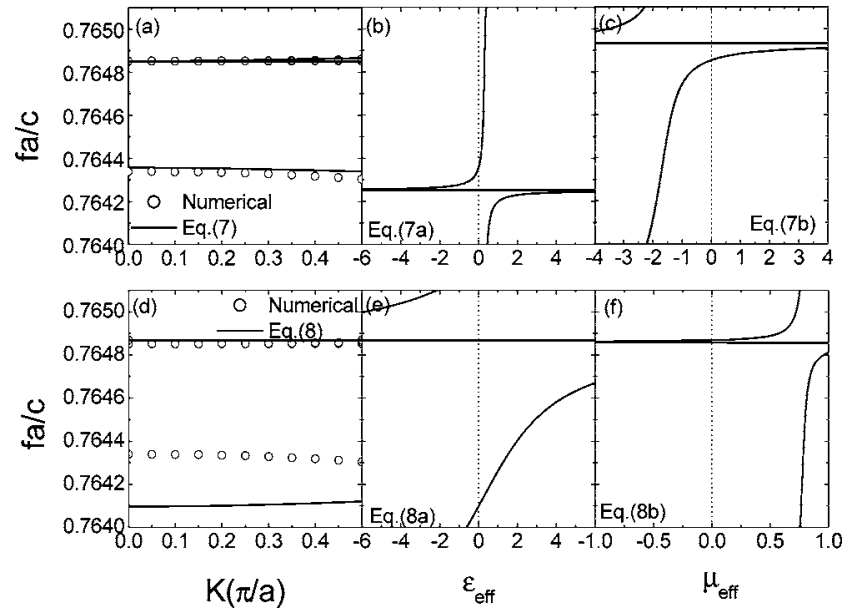

FIG. 10. (a) The circles denote the band structures near the $\Gamma$ point in the frequency range $0.7632 \leqslant \tilde{f} \leqslant 0.7645$. The solid curves represent the effective medium results of Eq. (7). (b) and (c). The effective permittivity and effective permeability obtained from Eq. (7), respectively. (d) and (e) show the same variables as (a)-(c) but all solid curves are obtained from Eq. (8).

real and imaginary parts of $n_{\text {eff }}(\tilde{f})$. It is interesting to note that the behaviors of the real and imaginary parts of $n_{\mathrm{eff}}(\tilde{f})$ near each pair shown in Fig. 9(a) resemble the behavior shown in Fig. 5(b). Thus, according to the previous description, we should expect a very narrow negative- $n$ band at $\tilde{f}$ $=0.76436$, where $\varepsilon_{\text {eff }}(\widetilde{f})=0$, due to the $m=0$ resonance. At $\tilde{f}=0.76485$, where $\mu_{\text {eff }}(\widetilde{f})=0$, there should exist a positive- $n$ band due to the $m=1$ resonance. Together with this band, there is a flat band due to the appearance of the longitudinal mode. All these predictions are confirmed by the bandstructure calculations, which are denoted by open circles in Fig. 10(a). The solid curves in Fig. 10(a) are constructed from $n_{\text {eff }}(\tilde{f})$ shown in Fig. 9(a). Again, we find excellent agreement between Eq. (7) and the band-structure calculations. This is also true for other resonances shown in Fig. 9(a). To show this, in Fig. 9, we mark the states at the $\Gamma$ point obtained from the band-structure calculations with solid squares for the $m=0$ states and open squares for the $m=1$ states. The positions of these states agree very well with the zeros of $n_{\text {eff }}(\tilde{f})$ in Fig. 9(a). For frequencies between $\widetilde{f}_{r 2}$ and 0.78 , Eq. (7) gives $n_{\text {eff }}(\widetilde{f}) \cong 0.725 i$, which is a pure imaginary number and, therefore, predicts a gap. Indeed, our bandstructure calculations do not give any states in this frequency region. The existence of this gap is due to a large negative value of $\varepsilon_{s}$, while $\mu_{s}$ is positive in this region.

Thus, our theory is quantitatively correct even when $\tilde{f}$ $=0.78$, which is equivalent to $k_{0} r_{0}=2.8$, or $\lambda_{0}=1.3 a$. When Eq. (8) is used, it is clear from Fig. 9(b) that the locations of zeros of $n_{\text {eff }}(\tilde{f})$ do not match the results of the band-structure calculations at the $\Gamma$ point. A more careful examination of Figs. 10(e) and 10(f) indicates that the lower band predicted by Eq. (8) is a positive- $n$ band as both $\varepsilon_{\text {eff }}(\widetilde{f})$ and $\mu_{\text {eff }}(\widetilde{f})$ are positive, whereas the higher band is a negative- $n$ band as both $\varepsilon_{\text {eff }}(\tilde{f})$ and $\mu_{\text {eff }}(\tilde{f})$ are negative. Both predictions are opposite to the results of the band-structure calculations. Thus, we have demonstrated unambiguously again that the region of validity of our effective medium theory goes beyond the long-wavelength limit.

Finally, we briefly discuss the flat bands shown in Fig. 3 near the frequency $\widetilde{f}_{s}=0.1536$. At this frequency, Eq. (17) gives $\mu_{s} / \mu_{0}=-1$, which is the resonance condition for magnetic surface plasmon in the cylinder. Thus, these nearly flat bands are caused by the weak coupling of the magnetic surface plasmon modes between the nearest-neighbor cylinders. They are located at $0.1527<\tilde{f}<0.1549$ at the $\Gamma$ point. In this frequency range, according to Eq. (7), we find $\varepsilon_{\text {eff }}(\tilde{f})>0$ and $\mu_{\text {eff }}(\widetilde{f})<0$ as shown in Figs. 4(b) and 4(c). Thus, our effective medium theory does not predict any states in this frequency range. The existence of flat bands must come from states with $m \geqslant 2$. In our band-structure calculations, we have found both pure states with single angular momentum index and mixed states involving two or more angular momentum indices. For simplicity, we show the case of pure states. In Fig. 11, we plot the electric field intensity maps in a unit cell for four states at the $\Gamma$ point: two $m=2$ states at $\tilde{f}$ $=0.152768$ and 0.154803 , and two $m=6$ states at $\tilde{f}$ $=0.153709$ and 0.153710 . It is clearly shown that all the wave functions are concentrated on the surface of the cylinder. The strong field localization is a result of sharp resonances in $D_{m}$ at $\tilde{f}_{s}$ when $m \geqslant 2$. This can be seen from an approximated form of $D_{m}$ near $\tilde{f}_{s}$, i.e., $D_{m} \cong i \beta_{m} /\left[\left(\tilde{f}-\tilde{f}_{s}\right)\right.$ $\left.+i \beta_{m}\right]$, where $\beta_{m}=2 \pi^{2 m+1} m\left(\tilde{f}_{s} r_{s} / a\right)^{2 m} /\left[(m !)^{2} \mu_{s}^{\prime}\left(\tilde{f}_{s}\right) / \mu_{0}\right]$ represents the width of the resonances. From Eq. (17), we can evaluate $\beta_{m}$ and find $\beta_{2} \approx 8.9 \times 10^{-6}$ and $\beta_{6} \approx 1.5 \times 10^{-18}$, which are indeed much smaller than $\tilde{f}_{s}$. The splitting in frequency for each pair is due to interaction between two nearest-neighbor cylinders. It is also seen that, for each pair of states, the intensity distributions are related by a rotation of $\pi / 2 \mathrm{~m}$. The lower frequency state is always accompanied by a high intensity along the $x$ or $y$ axis. The frequency splitting is smaller for a larger $m$. This is also expected because of stronger field localization as well as the smaller rotation required to go from one state to the other. Since only $m=0$ and 1 terms are considered in the effective medium formulas, the existence of these flat bands cannot be predicted by Eq. (7).

\section{CONCLUSION}

In summary, we have proposed here an effective medium theory for magnetodielectric composites. By comparing our theory with band-structure calculations on a periodic composite in two dimensions containing complicated electric and magnetic resonances in the microstructure, we show explicitly that while the long-wavelength theory fails when the wavelength in the background medium is smaller than 3.5 times the lattice constant, our theory is still valid even when 


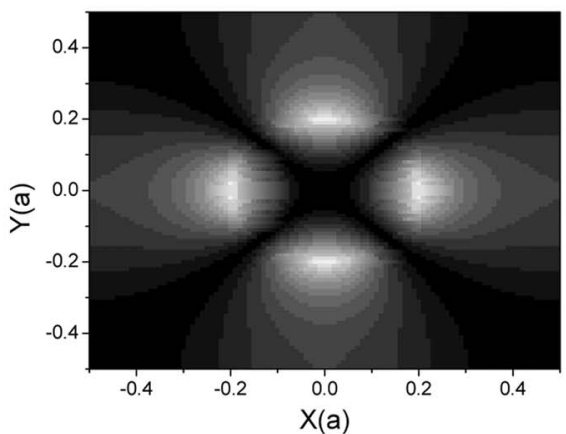

(a) $\mathrm{fa} / \mathrm{c}=0.152768$

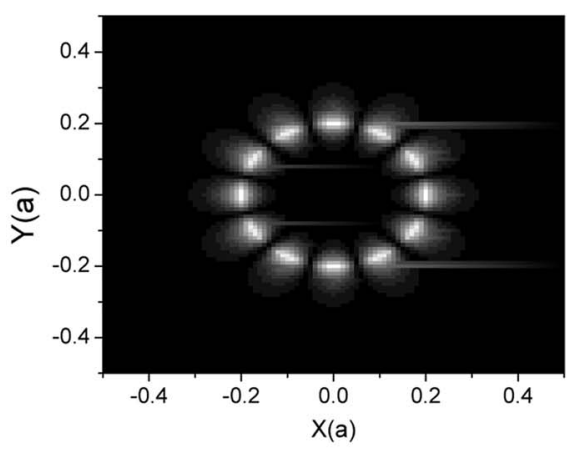

(c) $\mathrm{fa} / \mathrm{c}=0.153709$
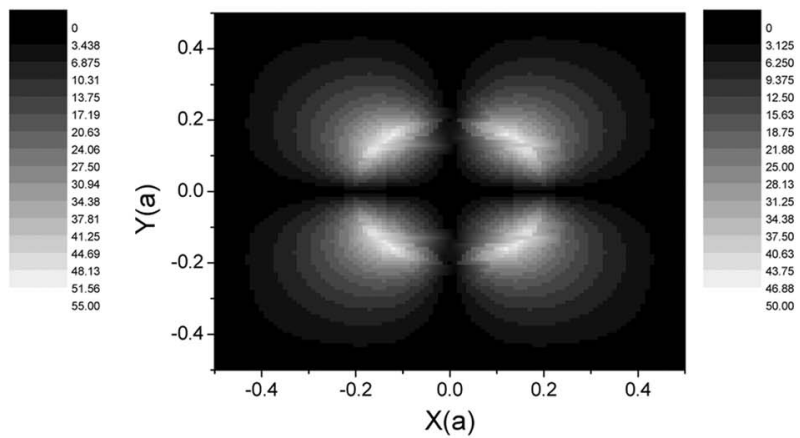

(b) $\mathrm{fa} / \mathrm{c}=0.154803$

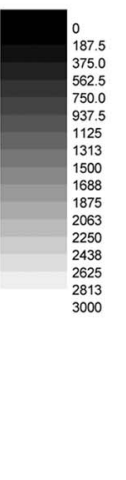

FIG. 11. Electric-field intensity distribution at different frequencies near $\tilde{f}=0.1536$.

the wavelength is as small as 1.3 times the lattice constant. Our theory reduces to the previous results when the longwavelength condition is imposed. Since our effective medium theory is obtained from the scattering of the $m=0$ and 1 components, it cannot predict those states with $m \geqslant 2$.

\section{ACKNOWLEDGMENTS}

The authors would like to thank Yun Lai for stimulating discussions. This work was supported by Hong Kong RGC Grant No. CA02/03.SC01.
${ }^{1}$ V. C. Veselago, Sov. Phys. Usp. 10, 509 (1968).

${ }^{2}$ D. R. Smith, W. J. Padilla, D. C. Vier, S. C. Nemat-Nasser, and S. Schultz, Phys. Rev. Lett. 84, 4184 (2000); R. A. Shelby, D. R. Smith, S. C. Nemat-Nasser, and S. Schultz, Appl. Phys. Lett. 78, 489 (2001); R. A. Shelby, D. R. Smith, and S. Schultz, Science 292, 77 (2001).

${ }^{3}$ J. B. Pendry, Phys. Rev. Lett. 85, 3966 (2000).

${ }^{4}$ J. B. Pendry, A. J. Holden, D. J. Robbins, and W. J. Stewart, IEEE Trans. Microwave Theory Tech. 47, 2075 (1999).

${ }^{5}$ J. B. Pendry, A. J. Holden, W. J. Stewart, and I. Youngs, Phys. Rev. Lett. 76, 4773 (1996).

${ }^{6}$ S. T. Chui and Liangbin Hu, Phys. Rev. B 65, 144407 (2002).

${ }^{7}$ A. N. Lagarkov et al., J. Magn. Magn. Mater. 258-259, 161 (2003); C. L. Holloway, IEEE Trans. Antennas Propag. 51, 2596 (2003).

${ }^{8}$ D. Stroud and F. P. Pan, Phys. Rev. B 17, 1602 (1978).

${ }^{9}$ A. P. Lagarkov, A. K. Sarychev, Y. R. Smychkovich, and A. P. Vinogradov, J. Electromagn. Waves Appl. 6, 1159 (1992).

${ }^{10}$ L. Lewin, Proc. Inst. Electr. Eng. 94, 65 (1947).

${ }^{11}$ W. Lamb, D. M. Wood, and N. W. Ashcroft, Phys. Rev. B 21, 2248 (1980).
${ }^{12}$ N. A. Khizhniak, Zh. Tekh. Fiz. 29, 604 (1959).

${ }^{13}$ E. Matagne, J. Phys. III 3, 509 (1993).

${ }^{14}$ G. D. Mahan, Phys. Rev. B 38, 9500 (1988).

${ }^{15}$ P. C. Waterman and N. E. Pedersen, J. Appl. Phys. 59, 2609 (1986).

${ }^{16}$ J. E. Sipe and J. Van Kranendonk, Phys. Rev. A 9, 1806 (1974).

${ }^{17}$ A. K. Sarychev, R. C. McPhedran, and V. M. Shalaev, Phys. Rev. B 62, 8531 (2000).

${ }^{18}$ Th. Koschny et al., Phys. Rev. B 71, 245105 (2005).

${ }^{19} \mathrm{P}$. Sheng, Introduction to Wave Scattering, Localization, and Mesoscopic Phenomena (Academic Press, San Diego 1995).

${ }^{20}$ L. Tsang, J. A. Kong, and K.-H. Ding, Scattering of Electromagnetic Waves: Theories and Applications (Wiley, New York, 2000).

${ }^{21}$ S. Tretyakov, Analytical Modeling in Applied Electromagnetics (Artech House, Boston 2003).

${ }^{22}$ J. Li, L. Zhou, C. T. Chan, and P. Sheng, Phys. Rev. Lett. 90, 083901 (2003).

${ }^{23}$ L. M. Li and Z. Q. Zhang, Phys. Rev. B 58, 9587 (1998).

${ }^{24}$ M. Notomi, Phys. Rev. B 62, 10696 (2000). 